\title{
Sciatic Nerve Injury Caused by a Stretching Exercise in a Trained Dancer
}

\author{
Ho Yong Shim, $\mathrm{MD}^{1}$, Oh Kyung Lim, $\mathrm{MD}^{1}$, Keun Hwan Bae, $\mathrm{MD}^{2}$, \\ Seok Min Park, $\mathrm{MD}^{3}$, Ju Kang Lee, $\mathrm{MD}^{1}$, Ki Deok Park, $\mathrm{MD}^{1}$ \\ ${ }^{1}$ Department of Rehabilitation Medicine, Michuhol Rehabilitation Center, Incheon; \\ ${ }^{2}$ Department of Rehabilitation Medicine, Gachon University of Medicine and Science, Incheon; \\ ${ }^{3}$ Department of Radiology, Incheon Imaging Diagnostic Center, Incheon, Korea
}

Sciatic nerve injury after stretching exercise is uncommon. We report a case of an 18-year-old female trained dancer who developed sciatic neuropathy primarily involving the tibial division after routine stretching exercise. The patient presented with dysesthesia and weakness of the right foot during dorsiflexion and plantarflexion. The mechanism of sciatic nerve injury could be thought as hyperstretching alone, not caused by both hyperstretching and compression. Electrodiagnostic tests and magnetic resonance imaging revealed evidence of the right sciatic neuropathy from the gluteal fold to the distal tibial area, and partial tear of the left hamstring origin and fluid collection between the left hamstring and ischium without left sciatic nerve injury. Recovery of motor weakness was obtained by continuous rehabilitation therapy and some evidence of axonal regeneration was obtained by follow-up electrodiagnostic testing performed at 3, 5, and 12 months after injury.

Keywords Sciatic nerve lesion, Lower extremity, Muscle stretching exercise

\section{INTRODUCTION}

The sciatic nerve is the largest nerve fiber in the human body and is rarely injured during sporting activities. The usual causes of sciatic nerve injury are fractures caused by motor vehicle accidents or falls, hip or thigh contusion, hip surgery, an intramuscular injection, and neoplasms [1]. Hyperstretching is an extremely rare

Received December 26, 2012; Accepted April 30, 2013

Corresponding author: Oh Kyung Lim

Department of Rehabilitation Medicine, Michuhol Rehabilitation Center, 21 Namdong-daero 774beon-gil, Namdong-gu, Incheon 405-760, Korea Tel: +82-32-460-8310, Fax: +82-32-460-3722, E-mail: oklim0928@yahoo.co.kr

(c) This is an open-access article distributed under the terms of the Creative Commons Attribution Non-Commercial License (http://creativecommons. org/licenses/by-nc/3.0) which permits unrestricted noncommercial use, distribution, and reproduction in any medium, provided the original work is properly cited.

Copyright $\odot 2013$ by Korean Academy of Rehabilitation Medicine mechanism of sciatic nerve injury. According to a study conducted in the United States, only one of 190 cases of peripheral nerve injuries during a 20 -year period was sciatic nerve injury [2]. Here we present an exceptional case of a trained dancer who injured a sciatic nerve after a stretching exercise.

\section{CASE REPORT}

An 18-year-old female patient who had performed dance training for one year presented with right foot drop and weak push-off on gait after a routine groin stretching exercise. Long adductor stretch with both legs apart and knees straight was performed while sitting to fully stretch the inner thigh muscles once anteroposteriorly and once laterally. The patient's dancing tutor helped her stretch by compressing her torso forward, which is the routine 
procedure. Immediately, after stretching, she felt a blunt pain and numbness, followed by weakness of both feet. Two hours later the weakness of left foot was improved, but pain and numbness had remained. She visited an orthopedic clinic and was treated with medication for pain control, non-steroidal anti-inflammatory drug-based lotion, use of hot packs, and neuromuscular stimulation for 3 months. The symptoms were not improved by treatment and she was referred to our rehabilitation clinic for electromyography. The patient was alert and cooperative. In her medical history, there was no systemic or neurologic disease including compression neuropathy in any extremity. Her family history was negative for peripheral nerve or neuromuscular disease.

Based on initial physical examination, using the Medical Research Council scale, strength was $4 / 5$ in right knee flexors, 5/5 in right knee extensors, $3 / 5$ in right ankle dorsiflexors, and 2/5 in right ankle plantarflexors. Deep tendon reflexes were $2+/ 4$ for bilateral quadriceps, $1+/ 4$ for the right Achilles, $2+/ 4$ for the left Achilles, and 2+/4 for bilateral elbow flexors. The Babinski sign was not pres- ent, and sensation was diminished to pinprick and light touch on the entire right lower limb below the knee. Position sense was normal. Tibial pulses were $2+$ bilaterally and capillary refill was normal. Little peripheral edema remained in the right lower leg. Commonly encountered systemic peripheral nerve disorders were ruled out by evaluation using thyroid studies, serum/urine electrophoresis, and a vitamin B12 assay.

Three months after the injury, electrodiagnostic nerve conduction studies were performed in both lower limbs. Sural sensory nerve action potentials (SNAP) were absent on the right side. The amplitude of compound muscle action potentials (CMAP) of the right deep peroneal nerve was within normal range, but its conduction velocity was lower than the left side. The amplitude of CMAP on the right tibial nerve was one-third lower than on the left side, implying incomplete tibial nerve injury (Tables 1,2 ). A needle electromyographic examination showed abnormal spontaneous activity in the form of positive sharp waves and fibrillation potentials with polyphasic motor unit configuration and reduced recruitment in muscles

Table 1. Results of sensory nerve conduction study of lower extremities 3 months after injury

\begin{tabular}{|c|c|c|c|c|c|c|c|}
\hline \multirow{2}{*}{ Stimulation nerve } & \multirow{2}{*}{ Recording site } & \multicolumn{3}{|c|}{ Latency (ms) } & \multicolumn{3}{|c|}{ Amplitude $(\mu \mathrm{V})$} \\
\hline & & 3 mo & $5 \mathrm{mo}$ & $12 \mathrm{mo}$ & $3 \mathrm{mo}$ & $5 \mathrm{mo}$ & $12 \mathrm{mo}$ \\
\hline Rt sural nerve & Ankle & NR & 1.75 & 2.25 & NR & 2.9 & 3.7 \\
\hline Lt sural nerve & Ankle & 2.20 & - & 2.00 & 13.6 & - & 15.5 \\
\hline Rt superficial peroneal & Ankle & 2.00 & 2.10 & 1.95 & 15.2 & 13.0 & 24.8 \\
\hline Lt superficial peroneal & Ankle & 1.75 & - & 1.80 & 14.0 & - & 14.1 \\
\hline
\end{tabular}

Rt, right; Lt, left; NR, no response.

Table 2. Results of motor nerve conduction study of lower extremities 3 months after injury

\begin{tabular}{|c|c|c|c|c|c|c|c|c|c|c|}
\hline \multirow{2}{*}{$\begin{array}{c}\text { Stimulation } \\
\text { nerve }\end{array}$} & \multirow{2}{*}{$\begin{array}{c}\text { Recording } \\
\text { site }\end{array}$} & \multicolumn{3}{|c|}{ Latency (ms) } & \multicolumn{3}{|c|}{ Amplitude $(\mu \mathrm{V})$} & \multicolumn{3}{|c|}{ Conduction velocity $(\mathrm{m} / \mathrm{s})$} \\
\hline & & $3 \mathrm{mo}$ & $5 \mathrm{mo}$ & $12 \mathrm{mo}$ & $3 \mathrm{mo}$ & $5 \mathrm{mo}$ & $12 \mathrm{mo}$ & $3 \mathrm{mo}$ & $5 \mathrm{mo}$ & $12 \mathrm{mo}$ \\
\hline \multicolumn{11}{|c|}{ Deep peroneal nerve } \\
\hline Rt ankle & \multirow{2}{*}{ Rt EDB } & 3.95 & 2.35 & 3.45 & 5.6 & 5.9 & 8.0 & \multirow{2}{*}{45.3} & \multirow{2}{*}{45.6} & \multirow{2}{*}{48.3} \\
\hline Rt fibular head & & 10.35 & 9.15 & 9.25 & 5.6 & 5.3 & 7.8 & & & \\
\hline Lt ankle & \multirow{2}{*}{ Lt EDB } & 4.75 & - & 4.05 & 5.4 & - & 7.7 & \multirow{2}{*}{54.5} & & \multirow{2}{*}{50.4} \\
\hline Lt fibular head & & 10.25 & - & 10.00 & 6.1 & - & 7.4 & & & \\
\hline \multicolumn{11}{|l|}{ Tibial nerve } \\
\hline Rt ankle & \multirow{2}{*}{ Rt AH } & 3.45 & 3.15 & 3.20 & 9.1 & 10.5 & 17.5 & \multirow{2}{*}{47.7} & \multirow{2}{*}{48.4} & \multirow{2}{*}{51.7} \\
\hline Rt knee & & 11.20 & 10.80 & 10.35 & 6.4 & 7.7 & 12.8 & & & \\
\hline Lt ankle & \multirow{2}{*}{$\mathrm{Lt} \mathrm{AH}$} & 3.40 & - & 3.15 & 32.0 & - & 31.6 & \multirow{2}{*}{52.5} & & \multirow{2}{*}{51.1} \\
\hline Lt knee & & 10.45 & - & 9.90 & 25.1 & - & 26.3 & & & \\
\hline
\end{tabular}

Rt, right; Lt, left; EDB, extensor digitorum brevis; AH, abductor halluces. 
innervated by the right sciatic nerve including the right long head of the biceps femoris, peroneus longus, semimembranosus, tibialis anterior, medial, and lateral gastrocnemius. The right gluteus maximus, quadriceps, tensor fascia lata, and paraspinal muscles were normal (Table 3).

Magnetic resonance imaging (MRI) studies of the lower legs and the lumbar area showed swelling and strong high signal intensity in the right sciatic nerve the entire way from the ischial spine to the proximal common peroneal nerve and the distal tibial nerve. Mild edema of the right proximal hamstring muscle suggested muscle sprain without definite fascial thickening or hematoma associated with the sprain. No definite mass lesion compressing or contacting the right sciatic nerve was found. Also, partial tear and fluid accumulation was seen at the left internal obturator muscle around the ischial spine, and avulsion tear was found at the left hamstring tendon origin on the ischial tuberosity of the left leg. However, the left sciatic nerve still contacted the above lesion, but was not enlarged or edematous (Fig. 1).

She was diagnosed with right sciatic neuropathy based on the above data. Conservative rehabilitation therapy, such as muscle strength exercise, transcutaneous electrical nerve stimulation, and hot pack was continued.

At 5 months post-injury, right sural SNAP were evoked
(Table 1). There was no interval change, except a measured strength $5 / 5$ in the right knee flexors. At 12 months, increased amplitude of CMAP of the right tibial nerve from 10.5 to $17.5 \mathrm{mV}$ showed axonal regeneration (Table 2). Also, strength was graded as 5/5 in right ankle dorsiflexors, which was indicative of full motor recovery and $4 / 5$ in right ankle plantarflexors. These findings provided evidence of axonal regeneration in right sciatic neuropathy mainly in the tibial division.

\section{DISCUSSION}

Sports related sciatic neuropathy is rarely reported because the sciatic nerve is large and wrapped around substantial muscles and ligaments, which provide stability. Peripheral nerve injuries are usually caused by traumatic accidents, such as motor vehicle accidents, penetrating trauma after stabbing incidents, gun-shots, and stretching or crushing injuries after falls [3]. One study reported $16(2.9 \%)$ sports-related nerve injuries among 534 peripheral nerve injuries over 16 years, with only $6.3 \%$ having sciatic nerve injury [3]. Krivickas and Wilbourn [2] reported two sports related sciatic neuropathy cases among 190 electrophysiologically proven peripheral nerve fiber injuries studied over 20 years. The possible mechanisms of sciatic nerve injury are laceration, contusion, com-

Table 3. Needle electromyography results 3 months after injury

\begin{tabular}{|c|c|c|c|c|c|c|c|c|}
\hline \multirow{2}{*}{ Muscle } & \multicolumn{4}{|c|}{ Spontaneous } & \multicolumn{3}{|c|}{ MUAP } & \multirow{2}{*}{$\begin{array}{c}\text { Recruitment } \\
\text { Pattern } \\
\end{array}$} \\
\hline & IA & Fib & PSW & Fasc & Amp & Dur & PPP & \\
\hline Rt. TA & $1+$ & $2+$ & $2+$ & None & $\mathrm{N}$ & $\mathrm{N}$ & + & Red \\
\hline Rt. GCM (lateral) & $1+$ & $3+$ & $3+$ & None & $\mathrm{N}$ & $\mathrm{N}$ & + & Red \\
\hline Rt. GCM (medial) & $1+$ & $3+$ & $3+$ & None & $\mathrm{N}$ & $\mathrm{N}$ & + & Red \\
\hline Rt. PL & $1+$ & $2+$ & $2+$ & None & $\mathrm{N}$ & $\mathrm{N}$ & + & Red \\
\hline Rt. BF (long) & $1+$ & $1+$ & $2+$ & None & $\mathrm{N}$ & + & + & Red \\
\hline Rt. SM & $1+$ & $1+$ & $2+$ & None & $\mathrm{N}$ & + & + & Red \\
\hline Rt. QC & $\mathrm{N}$ & None & None & None & $\mathrm{N}$ & $\mathrm{N}$ & $\mathrm{N}$ & $\mathrm{N}$ \\
\hline Rt. GM & $\mathrm{N}$ & None & None & None & $\mathrm{N}$ & $\mathrm{N}$ & $\mathrm{N}$ & $\mathrm{N}$ \\
\hline Rt. LUM PSP (L5-S1) & $\mathrm{N}$ & None & None & None & & & & $\mathrm{NC}$ \\
\hline Rt. LUM PSP (L4-5) & $\mathrm{N}$ & None & None & None & & & & $\mathrm{NC}$ \\
\hline Rt. LUM PSP (L3-4) & $\mathrm{N}$ & None & None & None & & & & $\mathrm{NC}$ \\
\hline
\end{tabular}

MUAP, motor unit action potential; IA, insertion activity; Fib, fibrillation; PSW, positive sharp wave; Fasc, fasciculation; Amp, amplitude; Dur, duration; PPP, poly phasic potential; TA, tibialis anterior muscle; GCM, gastrocnemius muscle; PL, peroneal longus muscle; BF, biceps femoris muscle; SM, semimembranosus muscle; QC, quadriceps muscle; GM, gluteus maximus muscle; LUM PSP, lumbar paraspinal muscle; N, normal; Rt, right; Lt, left; NC, not checkable; Red, reduced. 

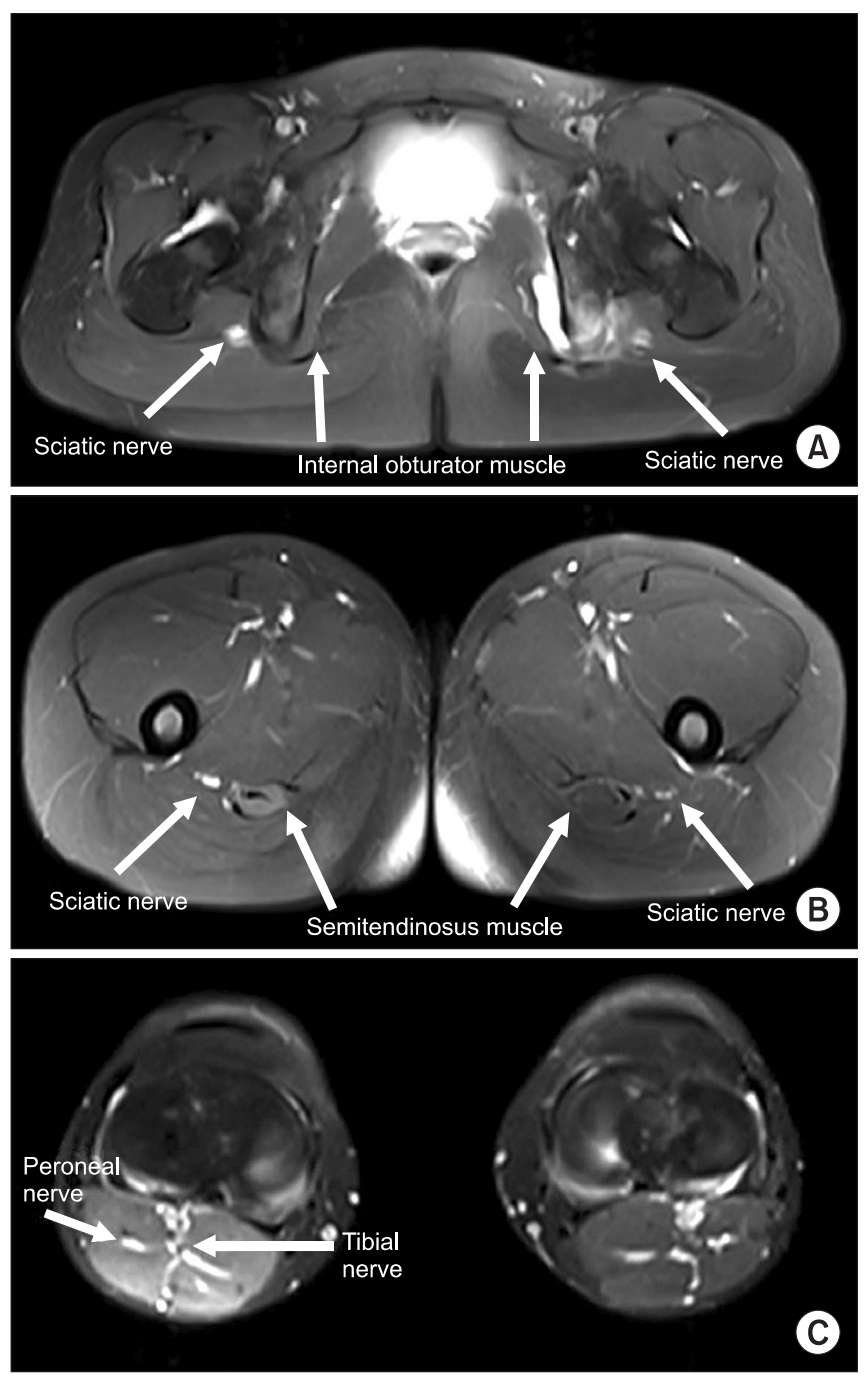

Fig. 1. FS T2 WI-axial scans. (A) FS T2 WI-axial scan at the level of the ischial spine shows stretching injury with abnormal high signal intensity in the right sciatic nerve, and left partial tear with high signal hemorrhagic fluid accumulation on left internal obturator muscle at insertion and the hamstring muscle origin. No definite mass lesion compressing or contacting the right sciatic nerve was found. Left sciatic nerve is contacting the above lesions, but not enlarged or edematous. (B) FS T2 WI-axial scan at the level of the mid femoral shaft shows continuous higher signal intensity through the right sciatic nerve, relative to left normal sciatic nerve with intermediate signal intensity. (C) FS T2 WI-axial scan at the level of the knee shows multiple denervation change with diffusely high signal intensity at the right distal biceps femoris muscle, right semitendinosus muscle, and both medial and lateral gastrocnemius muscles. FS, fat saturation; T2 WI, T2-weighted image. pression, ischemia, and hyperstretching. Most of the previous case reports concerning stretch related injury involved compression and hyperstretching over a long period. One reported domestic case of bilateral sciatic nerve palsy was caused by sleeping cross-legged position for 10 hours [4]. In a case from India, bilateral sciatic nerve compression occurred in a 42-year-old woman who took narcotics and sat with her torso bent forward and legs fully extended for 4 hours [5].

This case is significant because it shows the possibility of the sciatic nerve injury only with hyperstreching alone, compared to the other previous cases, which were commonly caused by hyperstretching and compression. Moreover, this case should be considered since it illustrates that even routine stretching exercise of short duration (30 minutes) can cause injury.

At 3 months post-injury, based on the nerve conduction test, conduction block was observed on left sural nerve and axonal injury was seen on right tibial nerve by side-to-side amplitude comparison (Tables 1, 2). Needle electromyographic investigation taken at 3 and 12 months revealed disappearance of denervation potentials, such as positive sharp waves and fibrillations, at 12 months post-injury, indicating Wallerian regeneration of the insulted nerve, right tibialis anterior muscle, peroneal longus muscle, biceps femoris muscle, and semimembranosus muscle (Table 3). According to the Seddon and Sunderland classifications, this case corresponds to type 2 axonotmesis, which is characterized by loss of nerve conduction at the site of injury in the distal direction and disruption of axonal continuity with Wallerian degeneration. The prognosis in type 2 axonotmesis is good because of intact endoneurium, which allows nerve regeneration in appropriate direction to the end organ [6]. In addition, the MRI findings showed widespread right sciatic nerve injury from ischial tuberosity to tibial nerve, supporting the clinical results (Fig. 1).

In summary, although the patient was accustomed to stretching exercise, the stretched sciatic nerve injured by traction and ischemic insult of minor degree. Thus, sufficient and stepwise warm-up training should precede exercise to prevent nerve, muscle, and tendon injuries.

\section{CONFLICT OF INTEREST}

No potential conflict of interest relevant to this article was reported. 


\section{REFERENCES}

1. Hyun JK, Lee SJ, Yoo DS, Park HG, Kwon BS. The electrodiagnostic findings of sciatic nerve injury according to the locations and etiologies. J Korean Acad Rehabil Med 2004;28:54-8.

2. Krivickas LS, Wilbourn AJ. Sports and peripheral nerve injuries: report of 190 injuries evaluated in a single electromyography laboratory. Muscle Nerve 1998;21:1092-4.

3. Kouyoumdjian JA. Peripheral nerve injuries: a retro- spective survey of 456 cases. Muscle Nerve 2006;34: 785-8.

4. Kwon IS, Bang HJ. A case report of bilateral sciatic neuropathy after sleeping with cross-legged position. J Korean Assoc EMG Electrodiagn Med 2007;9:189-92.

5. Walker M, Meekins G, Hu SC. Yoga neuropathy: a snoozer. Neurologist 2005;11:176-8.

6. Dumitru D, Amato AA, Zwarts MJ. Peripheral nervous system's reaction to injury. In: Electrodiagnostic medicine. 2nd ed. Philadelphia: Hanley \& Belfus; 2002. p. $115-58$. 